\title{
Physical examination: extended ok sign
}

\begin{abstract}
This article presents an elaboration of the widely used neurological examination OK sign, which by itself tests the anterior interosseous motor branch of the median nerve. However, the $\mathrm{OK}$ sign has the potential to be incorporated into an examination technique which tests several nerves simultaneously. The OK sign would continue to test the anterior interosseous nerve, whilst a simultaneous wrist extension additionally tests the radial nerve, metacarpophalangeal joint extension tests the posterior interosseous nerve, and abduction of the third to fifth digits tests the ulna nerve. This ensemble of manoeuvers has the ability to succinctly test the majority of motor nerves of the hand in a single smooth set of movements. As this multi-motor neurological test is a progression of the OK sign, the development has been called the 'Extended OK' sign; EOK.
\end{abstract}

Keywords: Physical examination, OK sign, Extended OK sign, EOK, Medial nerve, Radial nerve, Ulna nerve, Motor innervation, Hand
Volume 9 Issue 2 - 2017

\author{
Ali Abdullah Mohammed,' Fabienne Marie- \\ Louise Robertson, ${ }^{2}$ Nasir Shah ${ }^{3}$ \\ 'Arthroplasty Fellow, Department of Trauma and Orthopaedic, \\ Royal Liverpool University Hospital, UK \\ ${ }^{2}$ Senior House Officer, Department of Trauma and Orthopaedic, \\ Royal Liverpool University Hospital, UK \\ ${ }^{3}$ Orthopaedic Consultant, Department of Trauma and \\ Orthopaedic, Warrington and Halton Hospitals NHS \\ Foundation Trust, UK
}

Correspondence: Fabienne Marie-Louise Robertson, BA, MBBS, MPH, Senior House Officer, Department of Trauma and Orthopaedic, Royal Liverpool University Hospital, Prescot St, Liverpool, L69 3GA, UK, Email fabienne.robertson@gmail.com
Abbreviations: LOAF, Lumbricals, Opponens Pollicis, Abductor Pollicis Brevis, and Flexor Pollicis Brevis; EOK, Extended OK; MCPJ, Metacarpophalangeal Joint; PIPJ, Proximal Interphalangeal Joint; DIPJ, Distal Interphalangeal Joint

\section{Introduction}

A neurological examination of the upper limb can be grossly assessed through hand examination. Orthopaedic examination techniques illicit signs which inform the clinician about the integrity of motor and sensory nerves, as well as musculoskeletal function.

In general, the advent of investigations has greatly challenged the perceived need for physical examinations. ${ }^{1}$ However, modern thought is that history taking, physical examinations and investigations now form one sequential diagnostic entity, where one informs the other. ${ }^{1,2}$ A study by Peterson et al. ${ }^{3}$ echoed by Roshan, ${ }^{4}$ quantified the relative utility of each component of diagnosing and found that, in $76 \%$ of patients, history led the doctor to the correct diagnosis and that physical examination and investigations then served to increase confidence in that diagnosis. ${ }^{3,4}$

History taking guides physical examinations which in turn determines which investigations would best aid in further analysis of the patient's presentation. ${ }^{5}$ Using physical examination also prevents waste of needless investigations. ${ }^{5}$

Due to the nature of orthopaedics it is possible to readily illicit signs from the musculoskeletal system, and therefore physical examination skills are essential. Neurological upper limb examination reproducibility were determined to be moderate to excellent, and therefore demonstrated important diagnostic benefits. ${ }^{6}$

Therefore, although investigations may appear to overshadow physical examinations, in reality their utility remains essential in diagnostic practice. Therefore progress and the development of new and improved techniques should be encouraged and pursued.

\section{Anatomy review}

The hand is defined as the portion of the upper limb distal to the radiocarpal joint, and is composed of 27 bones, 14 of which are phalanges, 5 metacarpal bones, and 8 carpal bones.
Intrinsic and extrinsic muscles of the hand are responsible for the wide range of broad and intricate movements. The extrinsic muscles originate from the forearm whilst the intrinsic muscles originate and terminate within the compartment of the hand. Intrinsic muscles of the hand include: thenar, hypothenar, interosseous muscles (four dorsal, and three volar), and the lumbricals. The thenar eminence is found on the radial side of the palm, and is made up of three muscles: The abductor pollicis brevis superficially, which causes thumb abduction, flexor pollicis brevis which causes thumb flexion, and opponens pollicis which is deep to the abductor pollicis longus and facilitates opposition of the thumb. The hypothenar eminence lies on the ulna side of the palm and is made up of three muscles: Abductor digiti minimi, flexor digiti minimi which causes flexion of the fifth digit, and opponens digiti minimi. Palmaris brevis is sometimes considered to be part of the hypothenar, however it is so thin it cannot be distinguished from the three hypothenar muscles deep.

All hand muscles are innervated by three main nerves: Radial, ulna, and median. These branch out from the brachial plexus, which extends from the $\mathrm{C} 5$ to $\mathrm{T} 1$ spinal nerves.

The radial nerve innervates the posterior compartment of the arm, namely the medial and lateral heads of the triceps brachii, and the posterior osteofascial compartment of the forearm, including brachioradialis, aconeus, extensor carpi radialis longus. It then divides into deep and superficial branches, where the former becomes the posterior interosseous nerve, whilst the latter provides sensory information to the dorsum of the hand. The deep branch becomes the posterior interosseous nerve after the supinator and innervates the extensor muscles of the hand and wrist as well as abductor pollicis longus.

The median nerve enters the upper limb from the axilla past the inferior border of teres minor, then descends along with the brachial artery, starting laterally then crossing anteriorly to lie medial to the artery, on the medial side of the arm between the biceps brachii and brachialis. The nerve then passes anteriorly over the medial epicondyle into the cubital fossa medially, then continues into the forearm between the two heads of pronator teres, then between c, and finally emerges between the flexor digitorum superficialis and flexor pollicis longus. Starting proximally the median nerve innervates pronator 
teres, flexor carpi radialis, palmaris longus, and flexor digitorum superficialis. Just distal to the lateral epicondyle, the median nerve gives off the anterior interosseous nerve which innervates flexor pollicis longus, flexor digitorum profundus for the lateral second and third digits, and pronator quadratus. Finally, the medial nerve passes through the carpal tunnel on the volar aspect of the wrist, deep to the flexor retinaculum, and then divides into motor and sensory branches: The recurrent branch provides motor innervation to the muscles of the thenar compartment, and the digital cutaneous nerve divides into the common and proper palmar digital branches which provide sensation to lateral palmar aspect of the hand and the dorsum of the fingertips of the first to third digits including the lateral aspect of the fourth digit. Finally the recurrent nerve is responsible for the motor innervation of the thenar muscles (Opponens pollicis, Abductor pollicis brevis and Flexor pollicis brevis) and the first and second Lumbricals which flex the metacarpophalangeal joint and extends the interphalangeal joints. All four of these muscles innervated by the recurrent nerve are known as the LOAF muscles.

The ulnar nerve is derived from the $\mathrm{C} 8$ to $\mathrm{T} 1$ nerves of the brachial plexus. The nerve then descends posteromedially down the humerus and passes behind the medial epicondyle (cubital tunnel) after which it enters the anterior compartment of the forearm. As the nerve descends, alongside and inferior to the ulnar artery, it supplies flexor carpi ulnaris and the medial half of flexor digitorum profundus for the 4th and 5th digits. Before entering the palm of the hand, through the ulnar canal (Guyon's canal), the nerve divides into three branches: Muscular, palmar and dorsal branches of the ulnar nerve. Once the ulna nerve passes over the superficial retinaculum it gives off two branches, namely the superficial and deep branches of the ulnar nerve, where the former innervates palmaris brevis and provides sensation over the palmar aspect of the third and fourth digits, and the latter innervates adductor pollicis longus, third and fourth lumbricals, interosseous muscles, and the hypothenar muscles (flexor digiti minimi, abductor minimi and opponens digiti minimi).

To summarise, the motor functions of the hand are controlled by three main nerves: The radial nerve causes extension of the wrist and digits. Whilst the median nerve is responsible for abduction and flexion of the thumb, opposition, and first and second digit metacarpophalangeal flexion and interphalangeal flexion and extension, and third and fourth proximal interphalangeal joint flexion. Finally, the ulnar nerve is associated with thumb adduction and flexion, third and fourth metacarpophalangeal flexion and interphalangeal joint extension, and adduction and abduction of first to fourth digits.

\section{The extended OK sign}

A common test used in clinical practice is the OK sign which grossly verifies the integrity of the median nerve, and specifically tests the anterior interosseous nerve. In this study we propose an Extended OK (EOK) sign which builds upon the traditional OK sign, and subsequently involves the following: OK sign (flexed index, flexed thumb), extended wrist, extended metacarpophalangeal joints (MCPJ), extended proximal interphalangeal joint (PIPJ), extended distal interphalangeal joint (DIPJ) of the $3^{\text {rd }}, 4^{\text {th }}$ and $5^{\text {th }}$ digits, and abducted $3^{\text {rd }}, 4^{\text {th }}$ and $5^{\text {th }}$ digits (Figure 1 ).

As previously mentioned, motor innervation of hand muscles is supplied by the radial, ulna, median nerves. In addition to the initial OK sign, which tests the anterior interosseous branch of the median nerve, wrist extension then tests the integrity of the radial nerve, digit extension reflects the integrity of the radial nerve and extensors, whilst metacarpophalangeal extension reflects the posterior interosseous nerve function, and digit abduction reveals the function of the ulnar nerve.

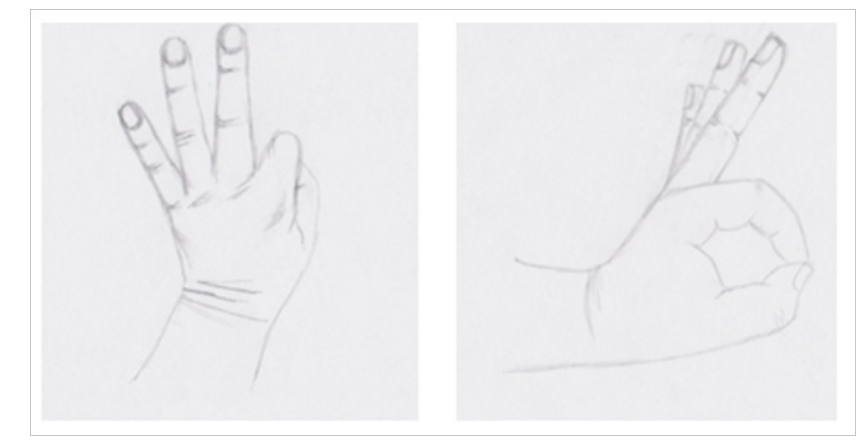

Figure I Physical Examination: Extended OK Sign-OK Sign, Extension Wrist and $3^{\text {rd }}-5^{\text {th }}$ Digits, Abduction of $3^{\text {rd }}-5^{\text {th }}$ Digits - Dorsal and Lateral Views.

The suggested order of examination is as follows:

a. OK sign ( $1^{\text {st }}$ and $2^{\text {nd }}$ digit flexion $)$

b. Wrist extension

c. Digit extension $\left(3^{\text {rd }} 4^{\text {th }} 5^{\text {th }}\right.$ MCPJ, PIPJ, DIPJ)

\section{d. Digit abduction $\left(3^{\text {rd }} 4^{\text {th }} 5^{\text {th }}\right.$ digits $)$.}

With the Extended OK sign, in one composite set of movements, upper limb motor nerve function is primarily tested.

\section{Discussion}

Specifically, extension of the wrist and digits tests the majority of the extensor tendons though does not specifically test the abductor pollicis longus. Therefore the integrity of the radial nerve is tested up to the level of the wrist and then more distally with the digits, but not medial innervation to the thumb. An extra examination for abductor pollicis longus would be needed to completely test the radial nerve.

The OK sign specifically tests for the anterior interosseous nerve which means it does not completely test for flexor digitorum superficialis, flexor digitorum profundus nor the LOAF muscles. More specific tests would be needed to complete examination of the median nerve for flexor digitorum superficialis, flexor digitorum profundus as well as the recurrent motor branch for the LOAF muscles.

Abduction of the digits is innervated by the deep ulna nerve which is a continuation of the ulna nerve. However, the function of the hypothenar muscles, medial lumbricals and adductor pollicis are not specifically tested and so further tests for the branches of the ulna nerve within the hand would need to be carried out to complete the examination of the motor ulna nerve.

The EOK examination is a screening tool and does not have the pretense of testing every digital branch of all three nerves and is not meant to provide a complete neurological assessment. Rather it has the ability to succinctly illicit potential motor deficits which will then require further examination and investigation.

General tests are taught to screen for possible hand injuries: Extension of the wrist and digits is taught to test the radial nerve, digit abduction as an indicator of ulna nerve function, and an intact OK sign demonstrates integrity of the median nerve. This EOK sign combines these basic principles into one succinct test, which in one move is able to generally test the motor innervation of the hand and reveal whether there exists a motor neurological deficit requiring further evaluation. 
At a time when clinicians are increasingly specialized and the role of non-doctor staff is developing, there is a need to establish sensitive tests which allow non-hand surgeons to screen patients for hand pathologies. Indeed, hand injuries are very common though the education and knowledge required to correctly diagnose hand pathologies is mostly restricted to orthopaedic and plastic surgeons. In view of this, proposing user-friendly physical examination tests which can illicit potential hand pathologies would address this gap of expertise and allow clinicians to screen for hand injuries in an efficient and straight forward manner. These latter characteristics of the examination, make the EOK sign also suitable for screening in trauma scenarios, multiple injury cases, and as an initial assessment of hand motor innervation. If an issue is identified by the EOK sign then further specific tests would be warranted.

\section{Conclusion}

The Extended OK sign is a straight forward evolution of the traditional OK sign offering an effective and efficient neurological screening test for the motor nerves of the hand. This test is sensitive for the majority of the three main hand motor nerves and would be useful in areas where time and/or expertise are limited.

At a time where investigations are the focus of innovation, it is important to highlight the continued significance and necessity to develop physical examination as an essential diagnostic tool in patient management. The Extended OK sign is a quick screening test for the radial, median, and ulna nerves which can be easily applied and taught.

I put it forward that, by adding three sequential movements to the widely used $\mathrm{OK}$ sign, namely wrist extension then metacarpophalangeal joint extension, followed by digit extension then abduction, the Extended OK sign is a logical clinical progression which has the ability to provide a quick and relatively simple general motor innervation screening test of the hand.

\section{Acknowledgements}

None to declare.

\section{Statement of funding}

This article received no specific grant from any funding agency in the public, commercial, or not-for-profit sectors.

\section{Conflicts of interest}

The authors declare that they have no conflict of interest.

\section{Statement of human and animal ethics}

This article does not contain any studies with human or animal subjects.

\section{References}

1. Abraham V, Blake C, Jerome PK, et al. Inadequacies of Physical Examination as a Cause of Medical Errors and Adverse Events: A Collection of Vignettes. Am J Med. 2015;128(12):1322-1324.e3.

2. Schultz MA, Doty M. Why the History and Physical Examination Still Matter. JAAPA. 2016;29(3):41-45.

3. Peterson MC, Holbrook JH, Von Hales D, et al. Contributions of the History, Physical Examination, and Laboratory Investigation in Making Medical Diagnoses. West J Med. 1992;156(2):163-165.

4. Roshan M, Rao AP. A Study of Relative Contributions of the History, Physical Examination and Investigations in Making Medical Diagnosis. J Assoc Physicians India. 200048(8):771-775.

5. Muhrer JC. The Importance of the History and Physical in Diagnosis. Nurse Pract. 2014;39(4):30-35.

6. Jepson JR, Laursen LH, Hagert CG, et al. Diagnostic Accuracy of the Neurological Upper Limb Examination I: Inter-Rater Reproducibility of Selected Findings and Patterns. BMC Neurology. 2006;6:8. 